\title{
La bactérie de la maladie de Whipple
}

La maladie de Whipple a été décrite en 1907 che\% un patient présentant un syndrome associant une diarrhée chronique, des épisodes d'arthrite, une pigmentation cutanée et un amaigrissement massif. A l'autopsie, des lésions cardiaques étaient observées, l'intestin grêle avait un aspect dysmorphique et on notait l'existence d'adénopathies mésentériques et d'une infiltration par des macrophages spumeux. D'autres symptômes ont depuis été attribués à cette maladic comme des atteintes du système nerveux central ou des endocardites parfois isolées et dont l'évolution peut être très sévère. Une origine métabolique de cette maladie a été évoquée jusqu'en 1961 où l'examen par microscopie électronique de l'intestin a révélé l'existence, dans le cytoplasme des macrophages, de bactéries de structure particulière puisqu'elles associent des éléments évocateurs de bactéries à Gram positif et d'autres de bactéries à Gram négatif. Cette découverte d'une origine bactérienne a permis depuis de traiter efficacement les patients atteints par antibiothérapie.

Le diagnostic de maladie de Whipple repose principalement sur l'étude des biopsies de l'intestin grêle, qui montre la présence caractéristique, dans les macrophages, de granules PAS (periodic acid-schiff) positifs qui correspondent à des bactéries intactes ou en dégradation. Au début des années 1990, Wilson et al. mettaient en évidence, sur les biopsies intestinales de patients, une séquence bactérienne d'ARN ribosomique $16 \mathrm{~S}$ [1]. L'analyse de cette séquence révélait alors que l'agent responsable de la maladie était vraisemblablement une bactérie à Gram positif, proche du groupe des actinomycieses et qui a été nommée Tropheryma whippelli par Relman et al. ([2] et $\mathrm{m} / \mathrm{s} 1992, n^{\circ} 8$, p. 869). Ia connaissance de cette séquence a permis de foumir un outil de diagnostic par PCR de la maladie.
Plus récemment, cette séquence d'ADN bactérien a aussi été détectée dans des biopsies jéjunales et dans le suc gastrique de patients n'ayant aucum signe de la maladie [3], ainsi que dans les eaux usées. Ces résultats suggèrent que la bactérie responsable de la maladie de Whipple, ou une bactérie très proche, est extrêmement répandue dans la nature voire présente dans la flore intestinale normale. La possibilité d'isoler Tropheryma whippelli représentait donc un atout essentiel, mais les nombreuses tentatives de mise en culture de cette bactérie se sont révélées infructueuses et, malgré les espoirs suscités par les résultats d'une équipe suisse [4], aucune souche disponible n'avait jusqu'à présent été obtenue. C'est maintenant chose faite puisque notre équipe vient d'isoler la souche bactérienne responsable de la maladie de Whipple [5].

Ce premier isolement a été effectué à partir d'une valve aortique prélevée chez un patient canadien qui présentait une endocardite isolée due à une maladie de Whipple. La bactéric a été cultivée en utilisant une lignée cellulaire de fibroblastes humains (HEL) et un milieu de culture dit "permissif ", ces conditions permettant en théorie de cultiver tous les microorganismes intracellulaires. Plusieurs facteurs ont permis cet isolement. Tout d'abord notre obstination puisque le premier effet cytopathique n'a été observé qu'après 65 jours de culture, mais aussi l'utilisation des cellules HEL qui vivent suffisamment longtemps après avoir cessé de se multiplier et qui permettent donc la croissance de cette bactérie, dont le temps de doublement est de 18 jours. Il s'agit en effet du temps de doublement le plus long observé pour une bactérie (celui de Mycobacterium leprae étant de 12 jours), ce qui explique probablement le caractère chronique de la maladic de Whipple. Nous avons pu ensuite cultiver cette bactérie de manière répétée, jusqu'à sept passages, et en obtenir une quantité suffisante pour déposer la souche au Centre national de culture des microorganismes. Cette culture nous a aussi permis de développer un test d'immunofluorescence indirecte très sensible mais dont la spécificité doit encore être améliorée. Enfin, grâce à la production d'anticorps spécifiques, la bactérie a pu être détectée par immuno-histochimie sur la valve aortique du patient.

Nous pensons ainsi avoir résolu une énigme qui avait commencé en 1907 et, en tout cas, ouvert la voie aux étapes suivantes dans la connaissance de Tropheryma whippelli et de la maladie dont elle est responsable. On peut en effet maintenant envisager l'obtention d'un modèle animal de cette maladie, le développement d'études génétiques ou de nouveaux tests sérologiques. Ces résultats soulignent en outre la nécessité de préserver un savoir-faire dans la culture des micro-organismes.

1. Wilson $\mathrm{kH}$, Blitchington $\mathrm{R}$, Frothingham $\mathrm{R}$, Wilson JA. Phylogeny of the Whipple's diseaseassociated bacterium. Lancel 1991; 338: 474-5. 2. Relman DA, Schmidt TM, MacDermott RP, Falkow S. Identification of the uncultured bacillus of W7ipple's disease. N Engl / Med 1992; 327: 294-301. 3. Ehrbar HU, Bauerfeind P, Dutly F, Koelz HR, Altwegg M. P('R-positive tests for Tropheryma whippelii in patients without Whipple's disease. Lancet 1999: 353: 2214

4. Schoedon G, Goldenberger D, Forrer R, et al. Deactivation of macrophages with interleukin-4 is the key (o) the isolation of Tropheryma whippelii. I In fect Dis 1997; 176: 672-7.

5. Raoult D, Birg MI., I a Scola B, el al. Cultivation of the bacillus of Whipple's diseasc. $N$ lingl J Med $2000 ; 342: 620-5$.

\section{Didier Raoult}

Unité des Rickettsies, Cnrs UPRESA 6020, 27, boulevard Jean-Moulin, 13385 Marseille, livance. 
Trypanosoma brucei synthétise ses propres acides gras : avantages et inconvénients. La forme circulante du trypanosome est couverte d'environ $10^{7}$ molécules identiques, une glycoprotéine du type GPI (glycosyl phosphatidylinositol) dont la fraction lipidique d'acides gras est composée de myristate. Le trypanosome a donc besoin de quantités très importantes de myristate. L'origine de ce myristate est un problème car cet acide gras n'est pas abondant dans les fluides biologiques de l'homme et on pensait que le parasite ne pouvait pas en produire. Faux: le trypanosome peut synthétiser du myristate par des réactions d'élongation à partir du laurate [1]. La synthétase d'acides gras n'est pas typique du monde eucaryote car elle produit du myristate plutôt que du palmitate, est associée à la membrane et est sensible à l'antibiotique thiolactomycinc. Cette voie de synthèse est unique car le myristate est préférentiellement incorporé dans la fraction GPI de cette glycoprotéine de surface et pas dans les phospholipides (qui sont formés d'une tête polaire et de deux acides gras hydrophobes). Comme la synthèse des GPI et des phospholipides a lieu dans le réticulum endoplasmique, il est raisonnable d'envisager que, che\% le trypanosome, la synthèse du myristate et son incorporation dans le GPI s'effectuent dans un endroit spécialisé du réticulum endoplasmique. L'incorporation du myristate dans le GPI est un événement important car cette réaction n'existe pas chez les mammifères et peut donc devenir une cible thérapeutique de la maladie du sommeil. Cette cible pourrait aussi être utilisée pour la malaria car le fait que la thiolactomycine inhibe aussi la croissance du Plasmodium falciparum suggère que cette voie de synthèse n'est pas spécifique du trypanosome.

[1. Morita YS, et al. Science 2000; 288: 140-3.]
Le phoque: réservoir naturel du virus de la grippe? Les infections par le virus de type B de la grippe (Influenıa B) semblent restreintes à l'homme, et l'on ne connaissait jusqu'à maintenant aucun réservoir naturel pour ce virus. Pourquoi pas les phoques? Pendant le printemps 1999, douze jeunes phoques ont été admis au SRCC (Seal Rehabilitation and Research Center) en Hollande pour des troubles respiratoires dont l'étiologie n'était due à aucune infection habituelle de cette espèce [1]. C'est à partir d'un prélèvement de gorge de l'un de ces phoques qu'un virus Influenza $B$ a été isolé. L'analyse de sa séquence révèle qu'il est extrêmement proche d'une souche ayant circulé chez l'homme quatre à cinq ans plus tôt. Cela suggère que cette souche humaine est à l'origine de l'infection puis de la propagation du virus dans la population de phoques depuis cette période. Cette hypothèse est confirmée par l'analyse sérologique et virologique rétrospective des sérums collectés chez plus de 900 phoques admis au SRCC montrant que, si aucun d'entre eux n'a été en contact avec le virus avant 1995 (année de l'infection humaine), $2 \%$ l'ont été depuis. La faible évolution de cette souche virale en quatre ans est inhabituelle et pourrait être due à la faible propagation du virus et à la plus longue durée d'infection observée chez les phoques. En conclusion, il apparaît que le phoque est un réservoir naturel de souches virales d'Influenza $B$ ayant infecté l'homme dans le passé. Pourrait-il être aussi à l'origine d'une réémergence de cette même souche chez l'homme?

[1. Osterhaus ADME, et al. Science 2000; 288: 1051-3.]
MCP-1, une chimiokine entre l'inné et l'acquis. MCP-1 (monocyte chemoattractant protein-1) est une chimiokine qui attire les monocytes aux sites d'inflammation. Dans les modèles d'athérosclérose et d'asthme par exemple, l'accumulation de macrophages dans les tissus lésés est liée à la production de MCP-1. Son expression est induite par des stimulus inflammatoires. Pour ces raisons, MCP-1 était considérée comme une chimiokine inflammatoire ayant peu d'effet sur l'immunité acquise et la migration des lymphocytes. Cette notion est maintenant à revoir, suite à la publication d'un article montrant le rôle du MCP-1 sur les lymphocytes T helper [1]. L.es deux grandes catégories de lymphocytes $\mathrm{T}$ helper (Thl et Th2) sécrètent des cytokines qui orientent la réponse immunitaire vers une immunité de type cellulaire (Th1) ou humorale (Th2). MCP-1 stimule la production d'interleukine 4 (cytokine Th2) et son expression exacerbée est associée à une mauvaise immunité cellulaire. Cela suggère que MCP-1 favorise les réponses immunitaires de type Th2; suggestion confirmée par l'observation des souris invalidées pour le gène MCP-1 qui ne peuvent plus produire de cytokines de type Th2. En conséquence, la commutation des sous-classes d'immunoglobulines n'a pas lieu et les souris sont résistantes aux infections par Leishmania major. En conclusion, MCP-1 influence à la fois l'immunité innée, par ses effets sur les monocytes, et l'immunité acquise en orientant la réponse immunitaire vers une immunité préférentiellement humorale.

[1. Gu L, et al. Nature 2000; 404: 407-11.] 